Supplement of Nat. Hazards Earth Syst. Sci., 20, 107-124, 2020

https://doi.org/10.5194/nhess-20-107-2020-supplement

(C) Author(s) 2020. This work is distributed under

the Creative Commons Attribution 4.0 License.

(c) (1)

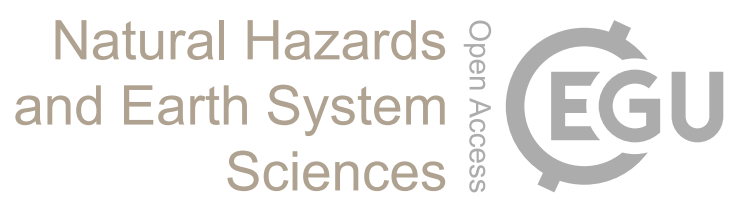

Supplement of

\title{
Improving sub-seasonal forecast skill of meteorological drought: a weather pattern approach
}

Doug Richardson et al.

Correspondence to: Doug Richardson (doug.richardson@csiro.au)

The copyright of individual parts of the supplement might differ from the CC BY 4.0 License. 


\section{Supporting Information}

\section{Markov model for weather pattern (WP) prediction}

Let $W(t)=i$ represent a particular WPi, with $i=1, \ldots, 30$, on day $t$. We construct a firstorder, nonhomogeneous Markov chain to predict WPs using the following procedure:

1. Calculate the 12 monthly transition matrices, $\mathbf{X}^{\boldsymbol{m}}, m=1, \ldots, 12$, of MO30:

$$
x_{i, j}^{(m)}=\operatorname{Pr}\left(W_{t}=i \mid W_{t-1}=j\right) \text {, for } i, j=1, \ldots, 30 .
$$

Each matrix is calculated using transition probabilities from three consecutive months, centred on the month of interest. For example, the transition matrix for June is calculated using data from May, June and July.

2. Set $W(t)$ as the observed WP at time $t=0$, the forecast initialisation day.

3. Generate a random number $x^{*}$ from the standard uniform distribution $U(0,1)$.

4. Find the index $y$ such that:

$$
\sum_{j=1}^{y-1} x_{i, j}^{(m)}<x^{*}<\sum_{j=1}^{y} x_{i, j}^{(m)}
$$

where $i=W(t)$.

5. Set $W(t+1)=y$ and $t=t+1$.

6. Repeat steps 3 to 5 until $t=T$, the final day to be forecast.

To provide probabilistic output, for each forecast we run 1000 Markov models in parallel. 


\section{Supplementary figures}

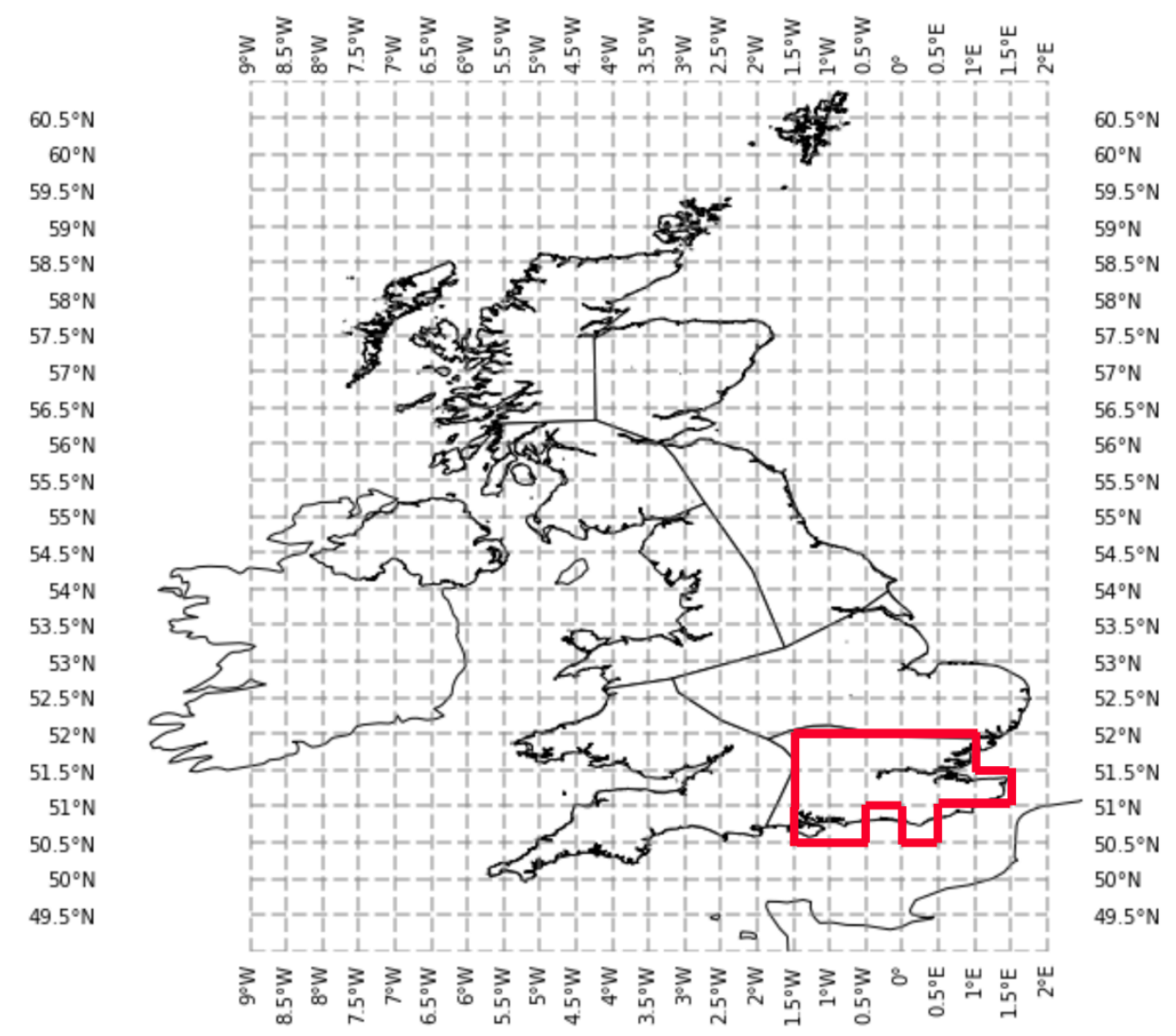

Figure S1: Schematic showing ECMWF-EPS precipitation forecast model grid, over the UK and HadUKP regions. The red box indicates the grid cells assigned to the region SEE using the cell centres. 

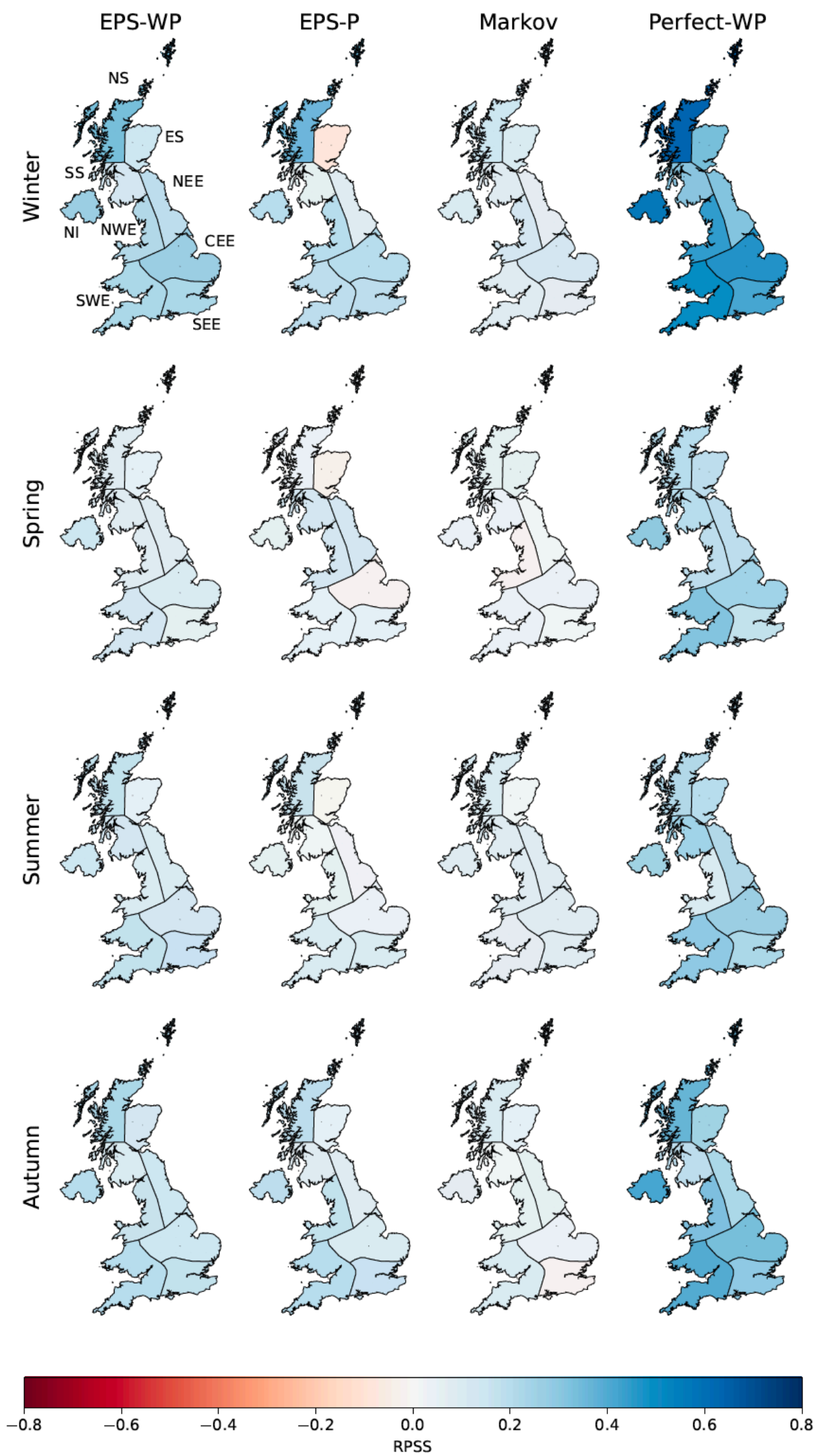

Figure S2: Ranked probability skill scores (RPSS) for precipitation forecasts at a 31-day lead for each model and season. 

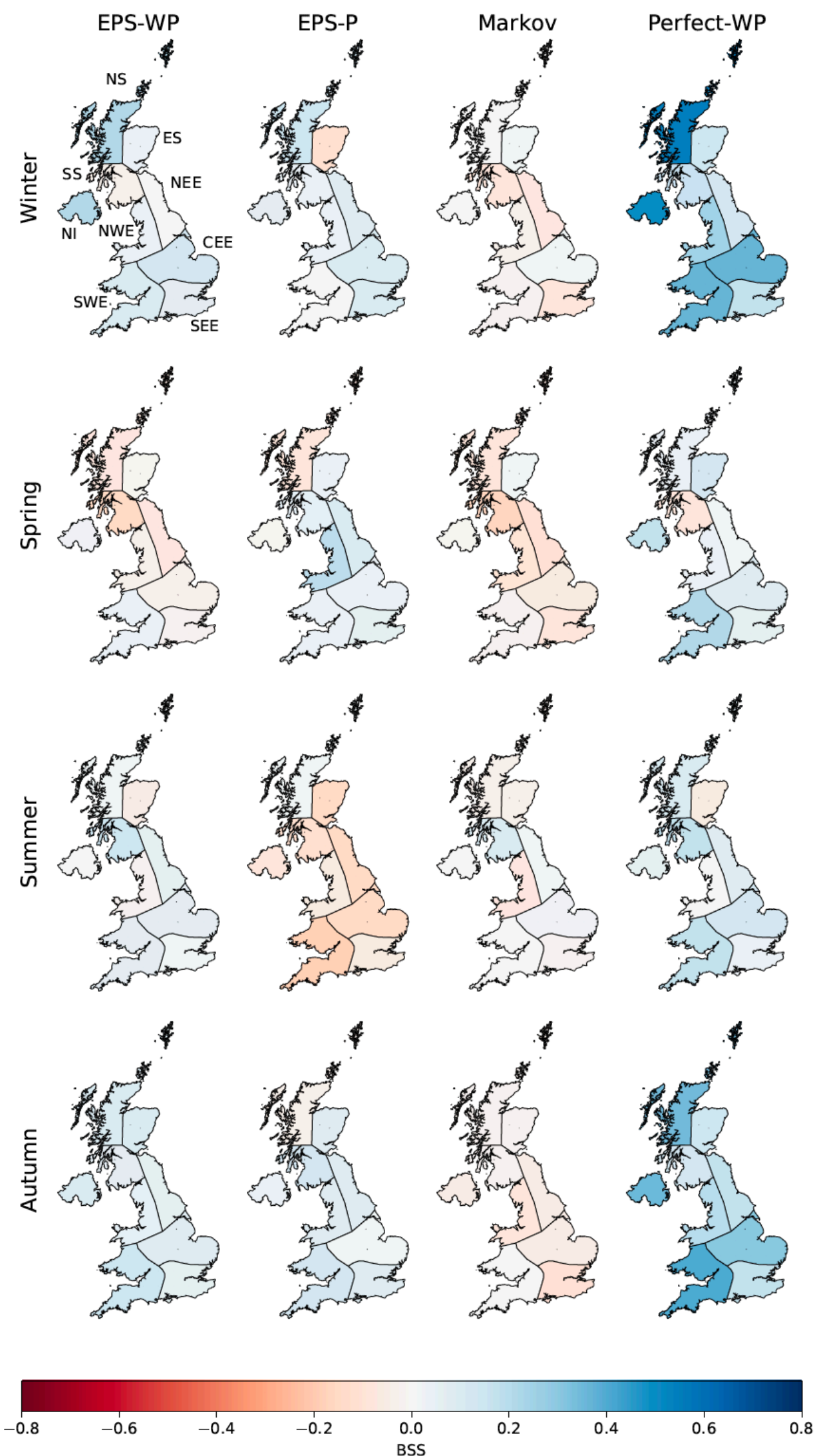

Figure S3: Brier skill scores (BSS) for mild drought (total precipitation below the 30.9th percentile) for a 31-day lead-time for each model and season. 